RAD Conference Proceedings, vol. 3, pp. 132-137, 2018

ISSN 2466-4626 (online) | DOI: 10.21175/RadProc.2018.29

www.rad-proceedings.org

\title{
mYOSL - A NEW SERIES OF PORTABLE AND STATIONARY EQUIPMENT FOR OSL-DOSIMETRY BASED ON BeO
}

\author{
D. Richter ${ }^{*}$, I. Słonecka ${ }^{2}$, S. Schischke ${ }^{3}$, K. Dornich ${ }^{1}$ \\ ${ }^{1}$ Freiberg Instruments GmbH, Freiberg, Germany \\ ${ }^{2}$ Central Laboratory for Radiological Protection, Warsaw, Poland \\ ${ }^{3}$ RadPro International GmbH, Wermelskirchen, Germany
}

\begin{abstract}
A new series of dosimetric devices, based on the optically stimulated luminescence (OSL) of BeO, is presented. The dosimetric properties of $\mathrm{BeO}$ and the use of OSL are favorable with a linear range at least up to $10 \mathrm{~Sv}$, near tissue energy response, and because of the use of OSL as a measurement technique, the dose information can be re-read. The myOSLraser device combines measurement and zeroing equipment in a single unit and is thus cost and time efficient. The manual reader for single myOSLdosimeters, consisting of two BeO-elements for Hp(o.o7) and $H p(10)$, can be expanded by myOSLautomation, which allows a fully automated analysis of 200 dosimeters. The system exceeds the requirements of EN/IEC 62387. A truly portable handheld device (myOSLchip) allows the manual operation of single element dosimeters, e.g. in phantoms. A simple method is proposed to use the OSL decay signal for the normalization of equipment and bringing into line the sensitivity between instruments.
\end{abstract}

Key words: Dosimetric equipment, myOSL, BeO, OSL-dosimetry, normalization of equipment

\section{INTRODUCTION}

In personal dosimetry, the difficulty in securing supplies of films for dosimetric purposes has caused the development of a personnel dosimetry system based on other materials and techniques. While thermoluminescence techniques are widespread, the use of Optically Stimulated Luminescence (OSL) has increased, with $\mathrm{Al}_{2} \mathrm{O}_{3}$ being a popular dosimetric material. We here present a new series of OSL equipment for dosimetric purposes (myOSL-series), which is based on $\mathrm{BeO}$ as an OSL-dosimeter because of the favorable properties of this material [1],[2]. Specifically, BeO exhibits a near tissue equivalency over a wide range of energies [3], which is favorable in personal dosimetry, while exhibiting similar properties in terms of the linearity of the dose-response, detection limits, etc. [4]-[7]. In OSL dosimetry only the first part of the signal is initially employed for analysis. In order to re-use dosimeters, the remaining signal has to be erased after reading, which is a time-consuming process when the built-in stimulation sources have to be used for that purpose. Therefore, such erasing (bleaching) is often performed in separate instrumentation.

The myOSLraser is a luminescence reader as well as an eraser in a single device, which is time- and costeffective. It is built for the use of 2-BeO-element ( $\mathrm{Hp}(0.07)$ and $\mathrm{Hp}(10)$, e.g. [8]) myOSLdosimeters.
For irradiation purposes the myOSLirradiator is equipped with a Sr-90/Y-90 source (37 MBq). Both devices are for manual single dosimeter use, but can both be extended with the myOSLautomation attachment, which is an automated feeding system for 200 myOSLdosimeters. Low weight, truly portable myOSLchip is used for manual OSL measurement of single BeO-element myOSLchip dosimeters. Measurement and data handing is achieved via the myOSLdosimetry software.

\section{THE MYOSL-SERIES}

While type testing for personnel dosimetry according to EN/IEC 62387 has been successfully completed, the systems are useful for and capable of other dosimetric purposes, like medical, accident and environmental dosimetry. Dosimeters for all myOSLsystems have individual numbers with the corresponding bar-code printed directly on the card holding the elements. This allows automated decoding by all systems and thus the required user interference is minimized.

\section{1. myOSLraser and myOSLdosimeters}

Efficient application in OSL dosimetry requires instrumentation capable of reading as well as fast zeroing of the OSL-signal remaining after reading. The reading, as well as the required zeroing is achieved in a single device (MyOSLraser, Figure 1) within 25

\footnotetext{
*daniel.richter@freiberginstruments.com
} 
seconds for a standard dose in personal dosimetry and thus no separate zeroing device is required. The OSL reading is performed by single $0.5 \mathrm{~s}$ stimulations of the elements with a blue LED. Stimulation light is cleaned by two GG420 and one KG5 filters, resulting in a stimulation wavelength of $\sim 460 \mathrm{~nm}$, which is close to the optimum of $435 \mathrm{~nm}$ for $\mathrm{BeO}$ [1]. The stimulation power is stabilized by a feedback loop with a photo diode [9]. Detection by a temperature stabilized Hamamatsu H10722-110 is restricted to a detection window around the dominant OSL luminescence emission of $\mathrm{BeO}$ at $370 \mathrm{~nm}$ [10] by $\mathrm{BP}_{3} 65 / 50$ and UG11 filters. The emission spectrum of the $\mathrm{BeO}$ used here was determined with the high sensitivity CCD-based spectrometry module of a lexsyg research luminescence reader [9]. The green stimulated OSL emission centers $\sim 375 \mathrm{~nm}$ wavelength (Figure 2), which is similar to [10].

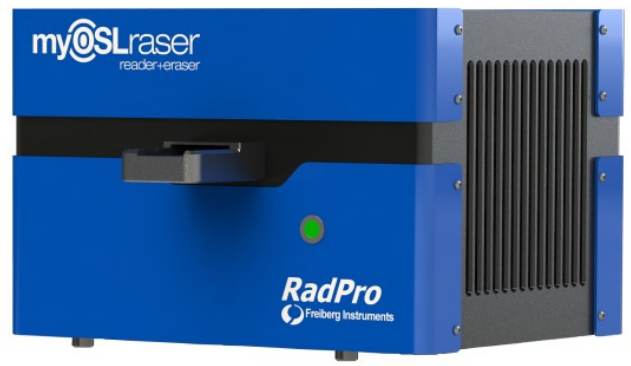

Figure 1. A myOSLraser device for single dosimeter use. The system reads the OSL and performs the required erasure of the remaining signal in a single instrument.

After OSL signal measurement, the remaining signal is erased by four separate high power blue LEDs simultaneously for both elements from both sides. This allows a fast and efficient procedure.

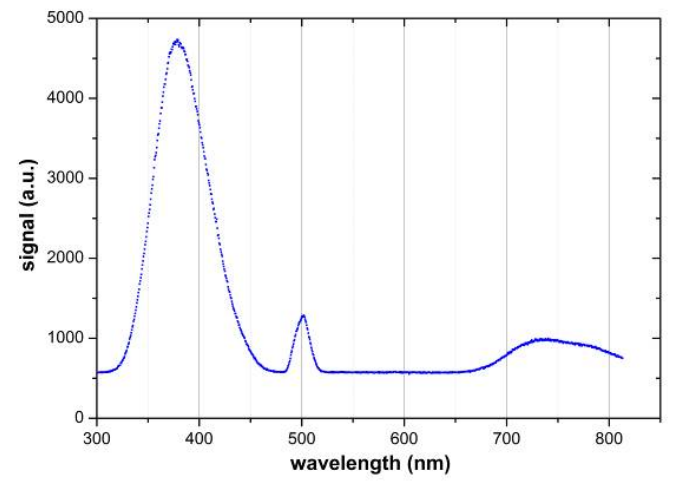

Figure 2. Emission spectrum of the first $20 \mathrm{~s}$ of green stimulated OSL from BeO (note the incomplete blocking of wavelengths $>480 \mathrm{~nm}$ by an edge filter results in a minor peak from the stimulation light).

In contrast to thermoluminescence, the dose dependent OSL-signal is not entirely erased by the reading procedure and it is thus possible to read the information again, which is a major advantage over many other passive dosimetry systems. As only a fraction of the dose information is removed, only a small correction is required for the signal results after re-reading, which allows a second dose determination. This also allows the accumulated use of dosimeters, where the previously measured dose has to be subtracted from the result every time.

The system provides excellent repeatability of the dosimetric material with coefficients of variation much less than $1 \%$ for a $1 \mathrm{mSv}$ dose. Such performance is achieved by the state of the art stabilization of luminescence stimulation and detection [10].

myOSLdosimeters (Figure 3) consist of 2-BeOelements for $\mathrm{Hp}(0.07)$ and $\mathrm{Hp}(10)$ made from Thermalox 995®, which can be read with the myOSLraser device. myOSLdosimeters exhibit an excellent linear dose response, a large dose range applicability and high sensitivity. Individual dosimeters are automatically identified by a bar-code within either system and managed for automated measurement, zeroing, irradiation, reconstruction of the history of a dosimeter, etc.

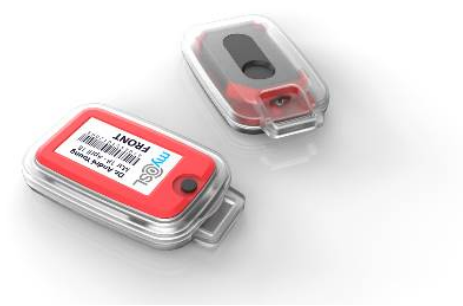

Figure 3. Both sides of a myOSLdosimeter holding two BeOelements for $\mathrm{Hp}(10) \& \mathrm{Hp}(0.07)$ in their case. A pin serves as an anti-tamper seal.

The dosimeters have dimensions of approximately $56 \times 34 \times 10 \mathrm{~mm}^{3}$ and are splash-water protected in a case made from polypropylene (PP) and polycarbonate (PC). The cases are equipped with labels showing dosimeter numbers, barcodes and names of allocated personnel. For opening the cover, a safety pin has to be broken and thus tampering can be detected.

$\mathrm{Hp}(10)$ is obtained for one element by a filter combination of $0.5 \mathrm{~mm} \mathrm{Al}$ plus $2.5 \mathrm{~mm}$ polycarbonate (PC). The energy response for $\mathrm{Hp}(10)$ is shown in Figure 4 and meets the requirements of EN/IEC 62387 with the application of a linear algorithm (factor 1.2).

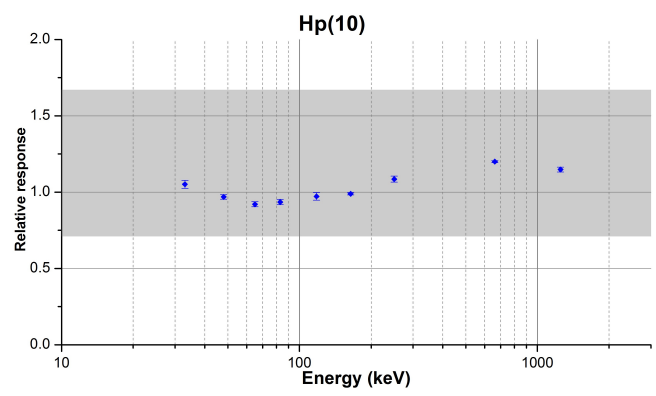

Figure 4. Energy response (with standard deviation; type A) of the myOSLdosimeter for $\mathrm{Hp}(10)$ after the application of a linear algorithm (1.2). 
The blue stimulated OSL lifetime was determined with the time-resolved ultra-fast pulsing module of a lexsyg research luminescence reader [9]. The measured lifetime of $25 \mu \mathrm{s}$ is in perfect agreement with the published data for $\mathrm{BeO}$ [11], [12].

\section{2. myOSLirradiator}

For irradiation purposes, e.g. local calibrations, the separate myOSLirradiator is equipped with a Sr-90/Y90 source $(37 \mathrm{MBq})$. The dosimeters are linearly moved back and forth under the $\beta$-source, which results in a more homogeneous irradiation compared to using a shutter. Irradiations can be performed as the user sets the time or dose if the irradiator has been calibrated. The dose rate is approximately $3 \mathrm{mSv} / \mathrm{min}$.

\section{3. myOSLautomatic}

The measurement of large numbers of dosimeters, e.g. in dosimetric services, is achieved with the myOSLautomation attachment (Figure 5). This automated feeding system for 10 cassettes with 20 myOSLdosimeters each can be mounted on any myOSLraser or any myOSLirradiator. The myOSLdosimetry software automatically displays and flags dosimeters on the screen and via messages whenever user interference might be required. However, in order to use the equipment efficiently, such dosimeters are skipped during the sequential measurements of several dosimeters, allowing the user to handle them at a later stage. This might be the case when the user-set limits of the maximum number of readout cycles, bleaching time, total lifetime dose, etc, are reached.

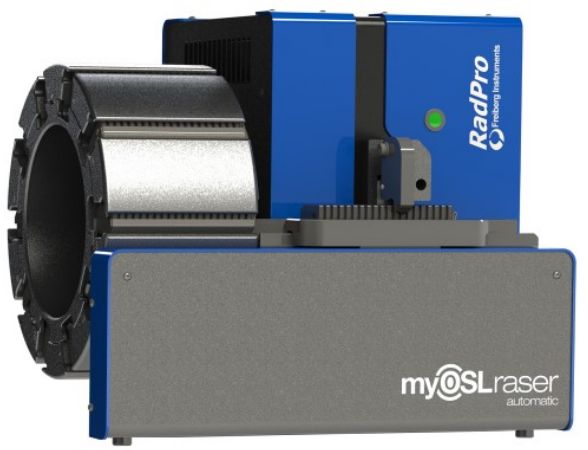

Figure 5. A myOSLraser OSL-reader/eraser with the myOSLautomatic feeder for 200 dosimeters.

\section{4. myOSLchip}

Flexibility is provided with myOSLchip - a single BeO-element OSL-reader (Figure 6). Due to its low weight, it is truly portable and can be even operated with battery packs. myOSLchip dosimeters (10x10 mm external dimensions) fit into standard phantoms and are bar-coded as well. Mechanical transport and positioning are achieved manually here. The user, therefore, has full control of the measurement and bleaching because these steps have to be performed individually and physically by turning a knob. Individual dosimeter calibration is possible here as well. The number of dosimeters and measurement data managed by the device itself is restricted only in portable mode, but the full use of the myOSLdosimetry software can be achieved in non-portable mode with the connection to a personal computer.

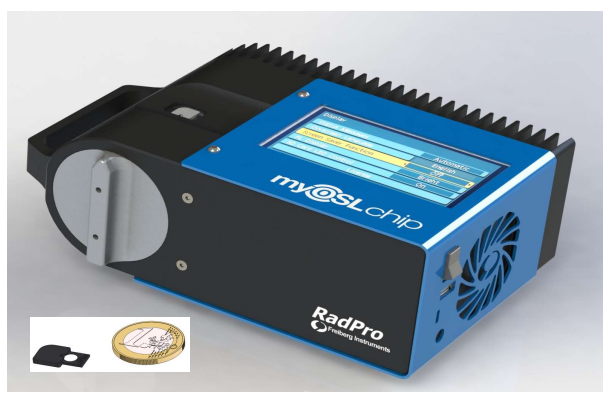

Figure 6. A myOSLchip device for single element BeO-OSL dosimetry. Dosimeters fit standard phantoms $\left(10 \times 10 \mathrm{~mm}^{2}\right)$. The device is truly portable and can be operated using power packs.

\section{5. myOSLdosimetry}

The myOSLdosimetry software allows full scale data management for any device of the myOSL-series, including an individual dosimeter as well as device calibration. Dosimeters exceeding user-defined single or total accumulated threshold doses, total bleaching time, number of measurement cycles, etc are automatically flagged and, if applicable, not measured in automatic mode. The user is informed on the required interference in detail by a message and each measurement, bleaching and irradiation step is recorded. A user-defined threshold of the maximum dose can be set. Above this threshold, the system does not automatically perform the required bleaching, which allows the manual re-measurement for verification.

Users can define individual personnel specific background levels and the maximum accepted background signal after bleaching can be set, as well as the maximum total accumulated time allowed for bleaching. For accumulated dose mode, where the remaining signal is not erased, it is advisable to define the maximum number of uses. Dummy dosimeters can be defined for myOSLautomation. They trigger the system to stop automated measurement from the position where the user has placed the dummy onward.

All data is maintained even if dosimeters are re-calibrated or otherwise changed, which allows the full reconstruction of the life history of a dosimeter. A full-scale database management is provided by myOSLdosimetry, including reporting functions, which allow the automated production of individual reports on myOSLdosimeter and also personnel histories with automated reporting functions.

The software is used to define dosimeters before use as calibration dosimeter (either externally irradiated by a SSD or with a myOSLirradiator), field dosimeter, quality control and dummy dosimeters. Designations can be changed if necessary and travel doses can be determined and subtracted. 


\section{MYOSLRASER AND MYOSLDOSIMETER PERFORMANCE}

Any luminescence measurement which is related to ionizing radiation includes uncertainties from irradiation and measurement. In the former, irradiation geometry (positioning) and timing is a major source of uncertainty, while in the latter readout geometry (positioning), stimulation power and its stability, dirt on stimulation and detection filter, as well as stability/sensitivity of the detector are of concern. Discerning the dominant source of uncertainty is therefore often not easy and the interpretation of the measurement results has to consider that often more than a single cause for observed variability has to be sought.

The myOSLraser is equipped with the possibility to monitor the functioning of the stabilization of stimulation power and detector stability by a short measurement of the stimulation LED light. It can be specified to be measured either before every element, before every dosimeter, every cassette or every $\mathrm{n}^{\text {th }}$ dosimeter. The resulting light level is stored with every record to document the stability of the system and can be assessed for analysis. The repeatability and reproducibility of the myOSLraser stimulation light and detection is depicted in Figure 7 , showing the stability of the system. A coefficient of variation of $0.7 \%$ over a 6-month period is obtained for the measurement of the stimulation light level before each element of the 384 dosimeters measured during this period, totalling 768 measurements (Figure 7 upper). A continuous run of 2156 myOSLdosimeters with myOSLautomat during the course of a day provides a coefficient of variation of $0.2 \%$ for these 4330 measurements (Figure 7 lower graph).

The dosimetric material together with the OSLequipment exhibits linear response for a wide dose range up to at least $10 \mathrm{~Sv}$ (Figure 8). The nominal detection limit is estimated as $\leq 0.05 \mathrm{mSv}$. By default a minimum waiting period for OSL measurement after irradiation of $15 \mathrm{~min}$ was applied here for all data presented.

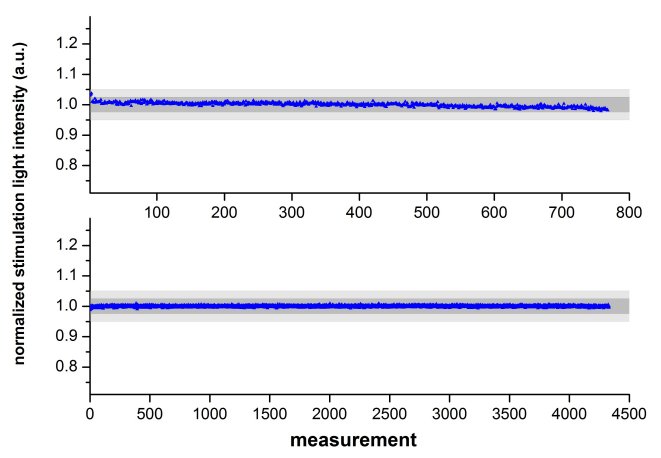

Figure 7. The repeatability and reproducibility of the myOSLraser stimulation light and detection (normalized to the average value) The dark shades represent the $2.5 \%$ and the light grey - the $5 \%$ variation range. Upper graph: over a 6month period (768 measurements); Lower graph: For 4330 measurements during a single day.

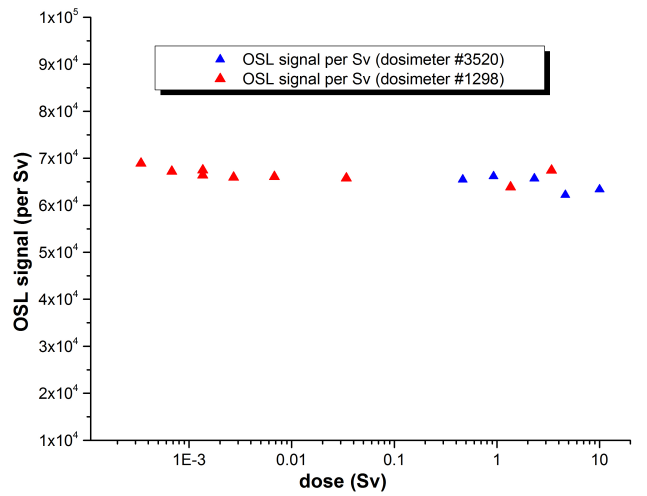

Figure 8 . The linearity of the $\beta$-dose response for single elements of two myOSLdosimeters is shown over a wide range of $\beta$-irradiations.

The repeatability of the myOSLraser is shown in Figure 19, where the variation of 5 cycles of exposure to the same dose followed by OSL-measurement (with a minimum of 15 min delay to avoid phosphorescence or other unstable signals) exhibits a small coefficient of variation which is below the limits set by EN/IEC 62387 and thus the myOSLraser is exceeding its requirements.

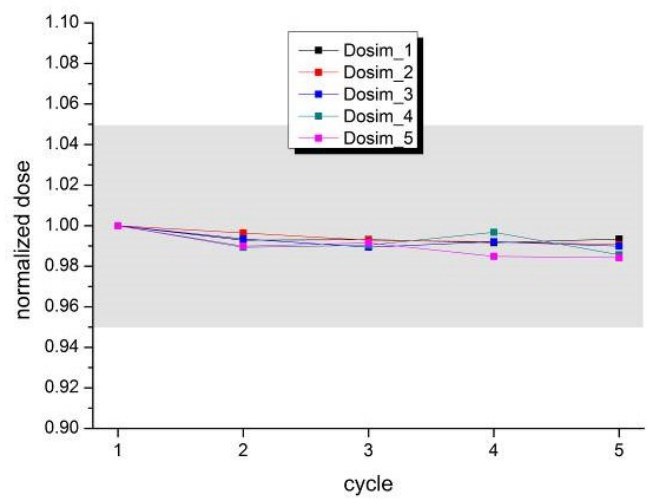

Figure 9. The repeatability of 5 cycles of $15 \mathrm{mSv} \beta$-irradiations with coefficients of variation way below 1 . The shaded area represents the acceptable 5\% range according EN/IEC62387.

The same experiment as in Figure 9 was performed for 24 randomly chosen dosimeters and the results indicated that an average coefficient of variation was 0.66 , with a maximum single value of 1.03 .

\section{THE PRINCIPLE FOR BRINGING DEVICE SENSITIVITIES BETWEEN INSTRUMENTS IN LINE}

Whenever several devices are employed to determine a quantity, it is convenient to have identical setups in order to achieve the same result, independent of the device used. Furthermore, potential problems can be easily identified by the measurement of a single dosimeter with different instruments, which have been exposed to the identical dose between measurements and thus should give the same answer. The accuracy of 
D. Richter et al., myOSL - equipment for OSL-dosimetry, RAD Conf. Proc., vol. 3, 2018, 132-137

the setups has to be questioned when deviation from the unity of such measurements is observed.

Employing comparable instruments in a laboratory and/or between laboratories is desirable. This requires devices which are nearly identical. The mechanical parameters of importance in the context of OSL detection are element/dosimeter positioning and distance of the element from the detector. They are not important given the precision of present day manufacture. The stimulation power is measured and set by the manufacturing. In the case of myOSLraser, the device is equipped with a loop for providing the required reproducibility and stability of stimulation power. The sensitivity of the luminescence detector (e.g. photomultiplier) is of concern. It has to be set within limits to obtain similar detection sensitivity. This is often achieved via a portable source which emits a stable light. However, it is often difficult to place a removable light source in an instrument in order to be able to perform such experiments under the actual geometric conditions of a dosimeter and at the same time with the electronics of the particular instrument. This is especially important when detectors have to be replaced and measurements have to be comparable before and after the replacement. The long-term stability of light sources has been questioned. LEDs and light sources based on radiofluorescence from the radioactive decay of isotopes with long half-lifes (e.g. C-14) provide sufficient stability [13].

However, the continuous wave OSL signal (cwOSL) provides the possibility for bringing in line luminescence detection devices entirely independent from any stable light source. Provided that the same detection sensitivity and the same stimulation power is employed, a combined series of relatively short cwOSL signals should follow the same signal decay as a single long cwOSL, which reduces the OSL-signal nearly to zero. If such short durations are set long enough (e.g. 3 s), so that an actual decay is observed, the following initial signal of the next measurement should match the previous last signal. The ratio of such light levels, which should be in practice as short as possible to avoid significant signal depletion (i.e $\leq 0.1 \mathrm{~s}$ ) can be used to adjust equipment and bring signal detection in line or even employed to quantify sensitivity difference, which eventually could be used for corrections. Obviously this is only applicable in the absence of signal fading over the required time span (Sommer 2013) and only applicable if any dose received after the irradiation is negligible.

To test the principle, 20 cwOSL signals from $\mathrm{BeO}$ elements with duration of 3 s were measured sequentially as separate measurements, but within a single instrument. This guarantees the identical measurement conditions and is intended to illustrate the principle. The light level of the last few channels (ms scale) of any $3 \mathrm{~s}$ cwOSL-signal should therefore always match those of the first few channels of the following stimulation. Furthermore, the OSL decay curve obtained when all 20 measurements are combined in a single dataset should be indistinguishable from a single cwOSL decay curve.

Figure 10 shows that 20 light levels match up very well and that the exponential fitting function of a single
$60 \mathrm{~s}$ cwOSL is indistinguishable from the combined data of the 20 cwOSL responses for the identical dose.

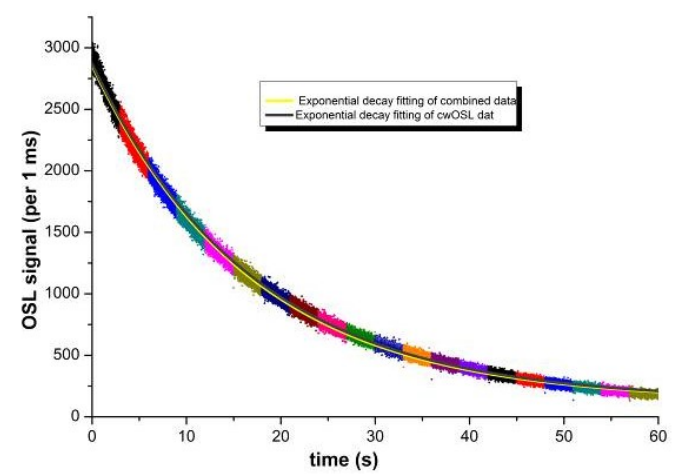

Figure 10. cwOSL measurements of a single BeO-element for device normalization. Various colors represent the $20 \mathrm{cwOSL}$ responses for sequential $3 \mathrm{~s}$ stimulation times. This data was combined to a single data set and fitted with a single decaying exponential (yellow line). The same fitting (grey line) is applied to a single $60 \mathrm{~s}$ cwOSL response for the identical dose.

The approach is thus shown to be feasible and it is a matter of choice and required degree of precision if only the light level matching and resulting ratios are employed, or mathematical functions are used to quantify the differences between luminescence detection systems.

While this approach does not necessarily have to result in a physical adaptation of a device, it could also be used as a means to determine a factor bringing in line the measured signals, if necessary.

\section{CONCLUSION}

The new myOSLraser dosimetric equipment, in conjunction with the two-element myOSLdosimeter ( $\mathrm{Hp}(0.07)$ and $\mathrm{Hp}(10)$ ) for BeO-OSL, exceeds the requirements of EN/IEC 62387. It is a versatile and fast set of devices, which provides a long linear dose response and high sensitivity. The luminescence properties of myOSLdosimeter are in agreement with the published values in terms of linearity and dose range, emission wavelength and lifetime. The separate attachment of myOSLautomation transforms the single dosimeter manual system into a fast automated device for 200 dosimeters, useful for dosimetric services. This automation also fits myOSLirradiator, which is equipped with a $\mathrm{Sr}-90 / \mathrm{Y}-90$ source (37 MBq). These devices are complemented by truly portable handheld myOSLchip, which allows the manual operation of single element dosimeters, e.g. in phantoms.

A method for using the OSL decay signal for the normalization of equipment and bringing into line the sensitivities between instruments is proposed.

Acknowledgements: The paper is a part of the research done within the project EP16014O funded by the German Ministry of Economy and Energy (BMWi) within the ZIM program. We thank David Sanderson 
for triggering the idea to use the OSL decay for device comparison.

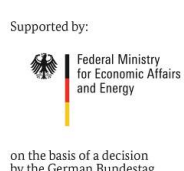

\section{REFERENCES}

1. E. Bulur, H. Y. Göksu, "OSL from BeO ceramics: new observations from an old material," Radiat. Meas., vol. 29, no. 6, pp. $639-650$, Dec. 1998 . DOI: $10.1016 / \mathrm{S} 1350-4487(98) 00084-5$

2. E. G. Yukihara, et al., "State of art: Optically stimulated luminescence dosimetry - Frontiers of future research," Radiat. Meas., vol. 71, pp. 15 - 24, Dec. 2014. DOI: 10.1016/j.radmeas.2014.03.023

3. A. J. J. Bos, "High sensitivity thermoluminescence dosimetry," Nucl. Instrum. Methods Phys. Res. B, vol. 184, no. 1-2, pp. 3 - 28, Sep. 2001. DOI: 10.1016/So168-583X(01)00717-0

4. D. P. Groppo, L. V. E. Caldas, "Luminescent response from $\mathrm{BeO}$ exposed to alpha, beta and X radiations," Radiat. Meas., vol. 71, pp. 81- 85, Dec. 2014. DOI: 10.1016/j.radmeas.2014.07.009

5. A. Jahn et al., "OSL efficiency for BeO OSL dosimeters," Radiat. Meas., vol. 71, pp. 104 - 107, Dec. 2014. DOI: 10.1016/j.radmeas.2014.03.024

6. A. Jahn et al., "Environmental dosimetry with the BeOSL personal dosimeter," Radiat. Prot. Dosimetry, vol. 170, no. 1-4, pp. 346 - 349, Sep. 2016.
DOI: $10.1093 / \mathrm{rpd} /$ ncwo79

PMid: 27060113

7. E. G. Yukihara, "BeO optically stimulated luminescence dosimetry using automated research readers," Radiat. Meas., vol. 94, pp. 27 - 34, Nov. 2016. DOI: 10.1016/j.radmeas.2016.08.008

8. S. Mattsson et al., "Dose Quantities and Units for Radiation Protection", in S. Mattsson, and C. Hoeschen Radiation Protection in Nuclear Medicine. Springer, Berlin, pp. 7-18

9. D. Richter et al., "Lexsyg - a new system for luminescence research," Geochronometria, vol. 40, no. 4, pp. $220-228$, Sep. 2013. DOI: $10.2478 / \mathrm{s} 13386-013-0110-0$

10. E. G. Yukihara, S. W. S. McKeever, Optically stimulated luminescence: Fundamentals and applications, Chichester, UK: John Wiley \& Sons Ltd., 2011.

DOI: 10.1002/9780470977064

11. E. Bulur, B. E. Saraç, "Time-resolved OSL studies on BeO ceramics," Radiat. Meas., vol. 59, pp. 129 - 138, Dec. 2013.

DOI: 10.1016/j.radmeas.2013.04.009

12. E. G. Yukihara, "Luminescence properties of $\mathrm{BeO}$ optically stimulated luminescence (OSL) detectors," Radiat. Meas., vol. 46, no. 6-7, pp. 580 - 587, Jun-Jul. 2011.

DOI: 10.1016/j.radmeas.2011.04.013

13. J. H. Dormo, Optical calibration of TLD readers, Trident Scholar Project Report No. 305., U.S. Naval Academy, Annapolis (MD), USA, 2003. Retrieved from: http://www.dtic.mil/dtic/tr/fulltext/u2/ a416350.pdf;

Retrieved on: Jun. 14, 2018 